La dimensión ambiental como componente de la educación superior, la Ciencia, y la tecnología en Cuba. PÁginas 83-96 en Revista de la Escuela de Ciencias de la Educación, año 10, número 9, enero a diciembre de 2014. ISSN 1851-6297. ISSN EN LINEA 2362-3349.

\title{
LA DIMENSIÓN AMBIENTAL COMO COMPONENTE DE LA EDUCACIÓN SUPERIOR, LA CIENCIA Y LA TECNOLOGÍA EN CUBA
}

\author{
Por Lourdes Ruiz Gutiérrez \\ (Instituto Superior de Tecnologías y Ciencias Aplicadas de La Habana \\ Universidad Internacional del Ecuador)*, Cuba. \\ lourruizgu@gmail.com
}

Recibido: 29/05/2014 Aceptado: 09/08/2014

\section{Resumen}

El presente trabajo plantea una síntesis histórica de la vinculación de la dimensión ambiental como componente de la educación, la ciencia y la tecnología en Cuba y expresa la relación directa de la investigación científica con la protección ambiental y el desarrollo sostenible como directiva de las políticas de la educación superior y científicas nacionales. Esta vinculación ha propiciado la aplicación de los nuevos conocimientos y resultados alcanzados y la evaluación del potencial de los recursos naturales existentes en el país, la educación ambiental por vías formales en todos los niveles de enseñanza y la educación comunitaria, la aplicación de prácticas productivas sostenible, el desarrollo de nuevas tecnologías para el aprovechamiento de residuales, incluidas las prácticas de producciones más limpias, la rehabilitación y restauración de ecosistemas afectados, la mejor gestión en el manejo de los recursos como agua y suelo, entre otros.

\section{Palabras Clave}

Ambiente - Educación superior - Ciencia - Tecnología.

\section{Abstract}

This paper presents a synthesis of the linkage of the environmental dimension as a component of education, science and technology in Cuba and expresses the

* Arquitecta, Máster en Arquitectura Tropical, Doctora en Ciencias Técnicas y Profesora jubilada del Instituto Superior de Tecnologías y Ciencias Aplicadas de la Habana y actualmente colabora como docente en la Universidad Internacional del Ecuador. Es miembro de la Organización del Tercer Mundo para Mujeres en las Ciencias y obtuvo el Premio de la Academia de Ciencias de Cuba y el Premio Especial en Medio Ambiente de la Ministra de Ciencias, Tecnología y Medio Ambiente de Cuba. 
Revista de la Escuela de Ciencias de la Educación, año 10, número 9, enero a diciembre de 2014. Páginas 83-96. ISSN 1851-6297. ISSN EN LINEA 2362-3349. LA DIMENSIÓN AMBIENTAL COMO COMPONENTE DE LA EDUCACIÓN SUPERIOR, LA CienCia y la teCnología en Cuba. Lourdes Ruiz GutiéRrez.

direct relationship of scientific research to environmental protection and sustainable development as a policy directive national higher education and scientific. This relationship has led to the application of new knowledge and achieved results and evaluation of the potential of the natural resources in the country, environmental -formal education at all levels of education and community education, the implementation of sustainable production practices the development of new technologies for the utilization of waste, including cleaner production practices, rehabilitation and restoration of affected ecosystems, better management in the management of resources such as water and soil, among others.

\section{Key Words}

Environment - Higher education - Science - Technology.

\section{Introducción}

El sistema educativo en la educación superior está estrechamente vinculado al sistema de ciencia, innovación tecnológica y medio ambiente de Cuba. Los mismos ejecutan la política científica y tecnológica nacional en todas las ramas de las ciencias y la innovación tecnológica a través de los centros investigación-desarrollo, y las universidades.

Se plantea por el Ministerio de Ciencia, Tecnología y Medio Ambiente (CITMA, 2005) que la política educativa y de ciencia, tecnología, innovación y medio ambiente de Cuba se plantea elevados retos en su proyección y se define por algunos de los siguientes principios:

- Garantizar la combinación de la educación superior con la investigación científica e innovación tecnológica, para que se revierta en un aporte a la sociedad y en estimular la reproducción del ciclo. Extender estos conceptos a la actividad científica de las universidades.

- $\quad$ Adoptar las medidas pertinentes para lograr la gestión integrada y efectiva del sistema de ciencia, tecnología, innovación y medio ambiente con la educación superior.

- Diseñar una política integral que tome en consideración la aceleración de sus procesos de cambio y creciente interrelación a fin de responder a las necesidades del desarrollo de la economía y la sociedad a corto, mediano y largo plazo.

En la dimensión ambiental, el sistema de ciencia e innovación tecnológica implementado en las universidades, formuló alternativas para mitigar o solucionar los problemas ambientales; sustentar, en forma armónica, racional y eficiente, el uso de los recursos naturales renovables y no renovables disponibles; controlar los problemas de contaminación, y viabilizar un desarrollo industrial basado en la producción de tecnologías autóctonas y la transferencia de tecnologías idóneas en función de las necesidades del país. 
Revista de la Escuela de Ciencias de la Educación, año 10, número 9, enero a diciembre de 2014. Páginas 83-96. ISSN 1851-6297. ISSN EN LÍNEA 2362-3349. LA DIMENSIÓN AMBIENTAL COMO COMPONENTE DE LA EDUCACIÓN SUPERIOR, LA Ciencia y la tecnología en Cuba. Lourdes Ruiz GutiérRez.

\section{Antecedentes de los problemas ambientales de Cuba}

Durante las cinco primeras décadas del pasado siglo veinte, se aceleró la devastación de los recursos naturales de Cuba. Predominaba una situación ambiental caracterizada por la degradación y erosión de los suelos, las aguas, el relieve y la vegetación. El área de bosques se había reducido hasta sólo $14 \%$ del territorio nacional, y se había producido una significativa pérdida de la biodiversidad. Después de 1959, las transformaciones económicas y sociales logradas por la Revolución Cubana, incidieron en una mejoría de las condiciones de vida de la población y en el incremento en las acciones de conservación de los recursos naturales, los que son considerados como patrimonio de todo el pueblo. Desde entonces, el gobierno de Cuba ha orientado una estrategia para elevar el nivel y la calidad de vida de la población, lo cual se ha combinado con diversas acciones, medidas y programas dirigidos a mejorar la situación ambiental. En la década de los años sesenta y en la primera mitad de los setenta, fueron creadas condiciones para el desarrollo de la ciencia, la educación y la salud. Los programas sociales, contribuyeron a reducir los problemas ambientales asociados a la pobreza, crearon capacidades institucionales y apoyaron la formación de potencial humano en la esfera ambiental (López, C, Iturralde, M, Claro, R, Ruiz, L, Cabrera, G, Molerio, L, 2002).

En el año 1962, fue la fundación de la Academia de Ciencias de Cuba, la cual consideró que la protección del medio ambiente siempre ha sido un área de desarrollo de la ciencia en Cuba y de la política educativa cubana de todos los niveles de enseñanza y para la población en general. A partir de la mencionada fecha, se crearon varios centros de investigaciones que abordan investigaciones sobre los recursos naturales y otras ramas en estrecha vinculación con las universidades. Se destaca el fortalecimiento del sistema educativo con la creación de institutos tecnológicos agropecuarios, universidades agrícolas, el Centro Nacional de Suelos y Fertilizantes y el Instituto de Suelos de la Academia de Ciencias de Cuba. También fueron creados otros institutos con perfiles afines, como los de Botánica, Zoología, Geografía y Oceanología, entre otros. Desde entonces, han surgido nuevas instituciones científicas con perfil marcadamente ambiental, a las que se unen otros centros de referencia como zoológicos, acuarios y jardines botánicos (Academia de Ciencias de Cuba, s.f.)

Durante la etapa de los años ochenta y noventa, se incrementó el número de instituciones de educación superior y de centros de investigaciones vinculados a las ciencias ambientales. Se promovió la formación científica, vinculada a estudios para un mejor conocimiento del medio ambiente, y a la investigación y desarrollo (I+D) en esa esfera. Se aplicaron resultados científicos en áreas clave del desarrollo sostenible, tales como la evaluación del potencial de recursos existentes, la aplicación de prácticas de agricultura sostenible, el desarrollo de vacunas y nuevos medicamentos, la generación de tecnologías para el aprovechamiento de residuales (incluidas las prácticas de producciones limpias), 
Revista de la Escuela de Ciencias de la Educación, año 10, número 9, enero a diciembre de 2014. Páginas 83-96. ISSN 1851-6297. ISSN EN LÍNEA 2362-3349. LA DIMENSIÓN AMBIENTAL COMO COMPONENTE DE LA EDUCACIÓN SUPERIOR, LA CienCia y la teCnología en Cuba. Lourdes Ruiz GutiéRrez.

la rehabilitación y restauración de ecosistemas afectados y una mejor gestión en el manejo de ecosistemas y recursos como el agua y los suelos, entre otros.

En el año 1992, Cuba participó en la Cumbre de la Tierra en Río de Janeiro, Brasil, y se firman los Convenios de Diversidad Biológica y Convención Marco de Naciones Unidas sobre el Cambio Climático. Entra en vigor el Convenio de Viena sobre la Protección de la Capa de Ozono y el Protocolo de Montreal. En el propio año, se modifica el Artículo 27 de la Constitución, para fortalecer la idea de la integración del medio ambiente con el desarrollo económico y social sostenible. Se introduce en el país el concepto de Desarrollo Sostenible y es implementada en 1993, la Agenda 21 de Cuba denominada como Programa Nacional de Medio Ambiente y Desarrollo, que se adecua en cada territorio del país.

Con la creación en 1994, del Ministerio de Ciencia, Tecnología y Medio Ambiente (CITMA), se produce una mayor institucionalización de la política y la gestión ambiental que determinó la actualización de los marcos estratégicos en materia de medio ambiente. En 1995 se organiza la Agencia de Medio Ambiente y sus centros de gestión ambiental y se formula el sistema jerárquico de regulaciones ambientales y dentro de la misma una prioridad la constituye la educación ambiental en estrecha colaboración con el Ministerio de Educación Superior, que es el organismo rector del sistema de educación superior cubano.

Con la aprobación de la Ley 81 del Medio Ambiente (1997) se mejoró el marco jurídico cubano en materia ambiental. La Ley 81 aborda entre otros temas, el de la educación para la gestión ambiental comunitaria y educación ambiental en todos los niveles de educación para promover el desarrollo sostenible y la protección del medio ambiente en las actividades económicas y sociales.

En 1997 se aprobó la Estrategia Ambiental Nacional (EAN) que profundizó las relaciones entre la educación, la investigación, el conocimiento científico, la innovación tecnológica, así como la protección y uso sostenible de los recursos naturales. La EAN constituye el basamento para el diseño e implementación de todas las Estrategias Ambientales Territoriales y de las Estrategias Ambientales Sectoriales, es un instrumento de trabajo para todas las provincias y sectores productivos del país. La mencionada estrategia ha sido actualizada sistemáticamente y en la misma se definen los principales problemas ambientales de Cuba que limitan el logro de la sostenibilidad ambiental y de manera crítica, se pueden mencionar los siguientes:

- Degradación de los suelos.

Los procesos erosivos afectan a 2,5 millones de hectáreas de suelos del país, el alto grado de acidez alcanza alrededor de 3,4 millones de ha, la elevada salinidad y sodicidad influencia alrededor de 1 millón de ha, la compactación incide sobre 2,5 millones de ha, los problemas de drenaje alcanzan 2,7 millones de ha. En definitiva, el $60 \%$ de la superficie del país se encuentra afectada por estos y otros factores (incluso por más de un factor a la vez) que pueden conducir a los procesos de desertificación (CITMA, 2007). 
Revista de la Escuela de Ciencias de la Educación, año 10, número 9, enero a diciembre de 2014. Páginas 83-96. ISSN 1851-6297. ISSN EN LÍNEA 2362-3349. LA DIMENSIÓN AMBIENTAL COMO COMPONENTE DE LA EDUCACIÓN SUPERIOR, LA CienCia y la tecnología en Cuba. Lourdes Ruiz GutiérRez.

- Afectaciones a la cobertura forestal.

Los incendios forestales son la causa mayor de afectación a los bosques en Cuba. Si bien en los últimos años se ha podido incrementar de forma constante la cubierta forestal, y en 43 años, el total de área cubierta del patrimonio forestal asciende a 2662 976,6 Mha, con un índice de boscosidad de 24,23\%, aún persisten las secuelas de años de explotación irracional de los bosques de Cuba, que prácticamente extinguieron valiosos recursos forestales (CITMA, 2007).

- Contaminación.

Existen diferentes causas que han motivado la contaminación de las aguas, los suelos y la atmósfera. Entre ellas, destaca la concentración de instalaciones industriales en zonas urbanas, lo que determina el empleo de las corrientes superficiales como receptoras de residuales crudos o parcialmente tratados, los que frecuentemente llegan a la zona costera. También influyen negativamente el empleo de tecnologías obsoletas, la indisciplina tecnológica, así como la no introducción de prácticas de Producción Más Limpia. Aún teniendo en cuenta los planes anuales de inversiones destinadas al medio ambiente, son insuficientes los recursos financieros destinados a la minimización, tratamiento, aprovechamiento, reuso de los residuos de la actividad agroalimentaria, industrial, del turismo, de hospitales y domésticos.

- Pérdida de la diversidad biológica.

Las características insulares del país, que han propiciado la evolución de una diversidad biológica particular y con valores muy altos de endemismo, condicionan a la vez la fragilidad y vulnerabilidad de algunos ecosistemas. Diversos procesos antrópicos han provocado la pérdida de biodiversidad en los ecosistemas frágiles como los arrecifes coralinos, manglares, las pluvisilvas y los bosques o matorrales remanentes de lo que fue la cobertura original de Cuba. Dentro de las causas principales que han provocado la pérdida de diversidad biológica se encuentra la fragmentación o destrucción de hábitat, ecosistemas y paisajes, debidos fundamentalmente al cambio del uso del suelo y al empleo de prácticas inadecuadas de pesca, cosecha y preparación de los suelos para la agricultura, entre otras.

- Carencia de agua.

Aun teniendo en cuenta el sustantivo desarrollo hidráulico cubano, que en algo más de cuarenta años ha posibilitado elevar nuestras capacidades de embalse de 48 a más de 9600 millones de m3, (CITMA, 2007) además de las obras de infraestructura para el uso de los recursos hídricos subterráneos, subsiste la carestía de agua para suplir todas las necesidades económicas sociales y ambientales, agravado esto por la ocurrencia de fenómenos naturales (sequía prolongadas, variaciones en el régimen estacional, etc.) y otros inducidos por causas antrópicas (intrusión salina, sobreexplotación, contaminación, etc.). 
Revista de la Escuela de Ciencias de la Educación, año 10, número 9, enero a diciembre de 2014. Páginas 83-96. ISSN 1851-6297. ISSN EN LÍNEA 2362-3349. LA DIMENSIÓN AMBIENTAL COMO COMPONENTE DE LA EDUCACIÓN SUPERIOR, LA Ciencia y la tecnologia en Cuba. Lourdes Ruiz GutiérRez.

La ejecución de la EAN (CITMA, 2007), promovió avances notables en la introducción de los enfoques de la sostenibilidad en Cuba dentro de los que se pueden mencionar los siguientes:

- Mayor conciencia social acerca de los problemas ambientales y mayor exigencia ciudadana por un estricto acatamiento de la legislación ambiental.

- Reordenamiento laboral en la montaña, caracterizado por un sesgo decisivo hacia los valores ambientales de estos ecosistemas, en particular, en lo referente a los bosques y el agua.

- Proceso de fortalecimiento paulatino del manejo integrado de la zona costera en su vínculo con el desarrollo económico-social, y la protección del medio ambiente.

- Resultados alcanzados por el Consejo Nacional de Cuencas Hidrográficas $(\mathrm{CNCH})$ en la gestión ambiental de las cuencas de interés nacional, que considera la cuenca como unidad de gestión ambiental para el desarrollo sostenible, con la aprobación de un número importante de programas vinculados con el aumento de la calidad de vida de la población y la protección de los recursos naturales.

- Los resultados obtenidos en el país acerca de las manifestaciones de la variabilidad climática y el cambio climático, las políticas de adaptación y mitigación, así como sus repercusiones sociales, económicas y ambientales.

\section{Experiencias en la esfera del medio ambiente y el desarrollo sostenible}

Las experiencias obtenidas en la ejecución de múltiples proyectos en la esfera del medio ambiente y el desarrollo sostenible y los ejercicios para una integración estratégica de la educación superior con la ciencia, la innovación tecnológica y el medio ambiente, han impuesto la actualización en diversos grados de sus respectivos marcos estratégicos que determinan dicha integración de las funciones.

Los territorios constituyen el principal escenario donde se materializan la política educativa, científica y ambiental. Los órganos de gobierno del Poder Popular, en estrecha interrelación con sus comunidades e instituciones educativas como las universidades, garantizan su aplicación en coordinación con los organismos de la dirección estatal a ese nivel para contribuir a la prevención y la eliminación de los principales problemas del medio ambiente en el país, lo cual incluye la erradicación paulatina de las insuficiencias detectadas en la aplicación de la política y la gestión ambiental.

Los lineamientos de la política económica y social del partido y la revolución en Cuba (PCC, 2011), plantean la necesidad de sostener y desarrollar investigaciones integrales en la educación superior en estrecha colaboración con los centros de investigación para proteger, conservar y rehabilitar el medio ambiente y adecuar la política ambiental a las nuevas proyecciones del entorno 
Revista de la Escuela de Ciencias de la Educación, año 10, número 9, enero a diciembre de 2014. Páginas 83-96. ISSN 1851-6297. ISSN EN LÍNEA 2362-3349. LA DIMENSIÓN AMBIENTAL COMO COMPONENTE DE LA EDUCACIÓN SUPERIOR, LA Ciencia y la tecnología en Cuba. Lourdes Ruiz GutiérRez.

económico y social, y en específico, promueven alcanzar un nivel superior en la protección del medio ambiente y el uso racional de los recursos naturales, con un uso eficaz de los recursos financieros y materiales de que dispone el país. Esto se materializa a través de las diversas formas de organización y asociación que se adoptan para el logro de fines sociales, culturales y científicos.

Para lograr una mayor coherencia en la operación del sistema se desarrollan diferentes instrumentos, tales como: los convenios de concertación de acciones con los Organismos de la Administración Central del Estado y las universidades; los acuerdos voluntarios para la mejor protección ambiental que se concretan con las principales empresas, dependencias, cooperativas, y otros; así como los códigos de conducta ambiental que se concretan en la implantación de buenas prácticas ambientales en las propias universidades las cuales sobresalen, por la cantidad de acciones y movilidad de actores (investigadores, académicos, personal calificado, etc.), con los Ministerios de Ciencia, Tecnología y Medio Ambiente, Salud Pública y Cultura entre otros. Ejemplo de esta vinculación se aprecia en las diversas provincias donde han surgido instituciones relevantes para la actividad ambiental, tales como la Universidad de Ciego de Ávila con el Centro de Investigación de Ecosistemas Costeros (CIEC), en Ciego de Ávila; la Universidad de Granma con el Instituto de Investigaciones Agropecuarias Jorge Dimitrov, en Granma; la Universidad de Oriente con el Centro Oriental de Ecosistemas y Biodiversidad (BIOECO), y el Centro de Investigaciones de Energía Solar (CIES) de Santiago de Cuba; la Universidad de Guantánamo el Centro de Investigación y Desarrollo de la Montaña, en Sabaneta, Guantánamo y el Centro de Investigación de Suelos Salinos, también en Guantánamo. A estas instituciones se adicionan otras muchas, vinculadas a actividades de producción y servicios, pero con un marcado perfil ambiental.

Algunas universidades claves en la enseñanza ambiental son: el Instituto Superior de Tecnologías y Ciencias Aplicadas (InSTEC), la Universidad de Pinar del Río (UPR), el Instituto Superior Politécnico "José Antonio Echeverría" (ISPJAE), la Universidad Agraria de La Habana (UNAH), la Universidad Central de Las Villas (UCLV), la Sede Universitaria de Sancti Spiritus (SUSS), la Universidad de Holguín "Oscar Lucero Moya" (UHOLM), la Universidad de Granma (UG), la Universidad de Oriente (UO), y sedes universitarias municipales, entre otras.

Resultan también relevantes las investigaciones que contribuyen a un mejor conocimiento del medio natural, mediante estudios e investigaciones afines que propician una acción más consciente sobre la biodiversidad y el medio ambiente. El desarrollo de las construcciones sostenibles y el turismo sostenible, dentro de las principales actividades económicas de Cuba se han estudiado en varias universidades cubanas. Algunos ejemplos son las investigaciones sobre la evaluación de impacto ambiental de las construcciones turísticas en la cayería norte y otras zonas costeras de Cuba (Ruiz, L. 1999) y 
Revista de la Escuela de Ciencias de la Educación, año 10, número 9, enero a diciembre de 2014. Páginas 83-96. ISSN 1851-6297. ISSN EN LÍNEA 2362-3349. LA DIMENSIÓN AMBIENTAL COMO COMPONENTE DE LA EDUCACIÓN SUPERIOR, LA CienCia y la teCnología en Cuba. Lourdes Ruiz GutiéRrez.

las tecnologías del concreto en su ciclo de vida (O’Reilly, V, Hernández, R. A. B, Gutiérrez, L. R, 2010), realizadas por el Instituto Superior Politécnico José Antonio Echeverría en coordinación con entidades del Ministerio de Ciencia, Tecnología y Medio Ambiente.

El desarrollo de un amplio sistema de salud centrado en el bienestar del hombre y en general de la comunidad, la sensible elevación del nivel de escolaridad de la población y de su calificación técnica profesional, constituyen elementos fundamentales de protección y conservación ambiental (PNUD, 2003).

La incorporación de la dimensión ambiental en los programas de desarrollo económico y social se realiza a través de diferentes vías y métodos educativos vinculados a los principales programas de desarrollo científicos del país, entre los que pueden mencionarse los siguientes:

- La colaboración de las universidades en la capacitación y elaboración de los programas y planes ambientales en las empresas productivas y municipios a partir de la agenda 21 o programa nacional de medio ambiente y desarrollo.

- La aplicación sistemática de los instrumentos de gestión ambiental incorporados en las diferentes carreras de pregrado y la educación de posgrados en las universidades.

- La cooperación, corresponsabilidad y coordinación de las acciones en torno a la protección ambiental, entre las comunidades, las universidades y los organismos productivos, de servicios y globales a nivel nacional y territorial.

Otra experiencia consiste en la incorporación de indicadores de desarrollo sostenible en las universidades que permiten organizar, sintetizar y elaborar la información, dando a conocer los objetivos y las metas de las políticas institucionales y son esenciales para la toma de decisiones bien informadas y en los procesos de planeación del desarrollo y del manejo ambiental (Gutiérrez, L, Céspedes, D. G, Cazorla, L. L, 2014).

Algunos ejemplos de los más importantes programas y proyectos científicos en la esfera ambiental y del desarrollo sostenible de Cuba (PNUD, 2003), donde han participado las instituciones de la educación superior, a lo largo de todo el país, son los siguientes:

- Programa de Bahías

Las bahías constituyen un elemento fundamental en la estructura, planificación y manejo de las costas de Cuba. También, desde el punto de vista social y económico, desempeñan un papel fundamental, si tenemos en cuenta que alrededor de ellas, es decir, en sus márgenes, se asientan las principales ciudades del país, se localizan los principales puertos y también las zonas industriales más importantes. A ello se agrega, que muchas de estas bahías, sostienen recursos pesqueros significativos que son explotados, así como actividades recreativas y turísticas. 
Revista de la Escuela de Ciencias de la Educación, año 10, número 9, enero a diciembre de 2014. Páginas 83-96. ISSN 1851-6297. ISSN EN LÍNEA 2362-3349. LA DIMENSIÓN AMBIENTAL COMO COMPONENTE DE LA EDUCACIÓN SUPERIOR, LA Ciencia y la tecnología en Cuba. Lourdes Ruiz Gutiérrez.

Por su importancia, desde hace algunos años, se trabaja en el diagnóstico y evaluación de la situación ambiental de las principales bahías cubanas (La Habana; Mariel; Matanzas; Cárdenas; Cienfuegos; Nuevitas; Puerto Padre; Holguín y Santiago de Cuba). Las principales acciones realizadas son:

- Identificación de los problemas existentes

- Inventario de los principales focos contaminantes y estimado de carga contaminante que llega a la bahía

- Evaluación de la calidad ambiental

- Identificación de alternativas de solución y sus costos

- Elaboración del Plan de Medidas requerido y Control de su cumplimiento

- Programa de Cuencas Hidrográficas

Se han definido 8 cuencas de máxima prioridad del país sobre la base de su complejidad económica, social y ambiental, el grado de afectación a sus recursos naturales y sus características generales. Estas ocho cuencas son: Cuyaguateje, Almendares -Vento, Ariguanabo, Zaza, Hanabanilla, Cauto, Toa y Guantánamo-Guaso, las cuales en su conjunto, abarcan una extensión de $15.000 \mathrm{Km}^{2}$ en el territorio de 11 provincias, albergan mas del $40 \%$ de la población cubana y alrededor del $60 \%$ de la actividad económica fundamental del país. Además, a nivel territorial se trabaja en las cuencas de interés provincial, que son 51 en total.

Las cuencas de interés nacional cuentan con sus correspondientes planes de acción, que han contribuido a la solución en algunos casos, y la mitigación en otros, de los problemas detectados. En estos planes, se destacan las acciones encaminadas a la reforestación, la actualización de los focos y reducción de la carga contaminante, así como las de educación ambiental y el mejoramiento de las condiciones higiénico-sanitarias de los asentamientos humanos, entre otras.

- Programa de Mejoramiento y Conservación de los Suelos

El programa contempla acciones dirigidas a la reducción y eliminación de las causas que provocan los procesos de degradación del suelo, tales como la erosión, la salinización, la acidez y la compactación, como fenómenos de mayor relevancia. Estas acciones tienen como objetivo:

- Incrementar la aplicación de medidas antierosivas temporales y permanentes

- Ejecutar medidas de acondicionamiento de los suelos

- Incrementar los niveles de ejecución de drenaje

- Aplicar enmiendas orgánicas y minerales; aplicar abonos verdes.

- Monitorear la calidad del agua para el riego.

- Incorporar la educación ambiental y la capacitación de los agricultores y pobladores. 
Revista de la Escuela de Ciencias de la Educación, año 10, número 9, enero a diciembre de 2014. Páginas 83-96. ISSN 1851-6297. ISSN EN LÍNEA 2362-3349. LA DIMENSIÓN AMBIENTAL COMO COMPONENTE DE LA EDUCACIÓN SUPERIOR, LA Ciencia y la tecnologia en Cuba. Lourdes Ruiz GutiérRez.

- Programa Nacional de Acción de Lucha Contra la Desertificación y la Sequía

Tiene como objetivo la ejecución de las acciones necesarias para detener y evitar estos procesos en el territorio nacional. Además responde a uno de los compromisos adquiridos por Cuba al ratificar, en el año 1997, la Convención Internacional de Lucha contra la Desertificación y la Sequía. El Programa contempla el desarrollo económico social de las zonas afectadas; la elaboración o adecuación de instrumentos jurídicos, políticas y estrategias; la educación y participación ciudadana; la investigación científica e innovación tecnológica; el fortalecimiento institucional; y la cooperación internacional, además se vincula con otros convenios como el de Diversidad Biológica y la Convención Marco de Naciones Unidas sobre Cambio Climático.

Dentro de las medidas que se consideran en el Programa se destacan las relacionadas con:

- La prevención de desastres naturales y antrópicos, tales como la sequía, los incendios forestales, los procesos de degradación de suelos, la deforestación.

- $\quad$ La recuperación y rehabilitación de suelos, la preservación de la calidad del agua y el uso sostenible de los recursos naturales.

- El mejoramiento de la calidad de vida de la población de las zonas afectadas tales como el incremento y calidad de los servicios básicos de salud, educación, servicio de agua potable y alcantarillado, y energía.

- $\quad$ Proyecto Internacional sobre Protección de la Biodiversidad y el Desarrollo Sostenible en el Archipiélago Sabana Camaguey

Con métodos participativos interdisciplinarios, intersectoriales e interterritoriales (320 investigadores y técnicos de universidades, 12 organismos de la administración central del estado y 5 provincias), mediante un enfoque de solución de problemas, se elaboró un plan estratégico dirigido a la protección de la biodiversidad y el avance hacia el desarrollo sostenible. La estrategia se basó fundamentalmente, en el establecimiento del manejo integrado costero, un sistema de áreas protegidas, una red de laboratorios de monitoreo ambiental y la aplicación de enfoques sostenibles en el planeamiento, diseño y desarrollo económicos.

También se efectuó, sobre bases ambientales, el planeamiento estratégico del desarrollo turístico y de protección de la biodiversidad de los cayos Santa María, Guillermo, Coco y Sabinal. Estos resultados se encuentran en un sistema de información geográfica, que constituye una poderosa herramienta en la adopción de decisiones para el manejo y desarrollo económico.

- Proyecto Internacional Sistema Integrado de Evaluación de la Sostenibilidad en Cuba.

Este proyecto reafirma el interés en la República de Cuba y su comunidad científica, de avanzar en una evaluación de los análisis de sostenibilidad, enfatizando en los límites físicos y naturales que lo enmarcan a nivel de país 
Revista de la Escuela de Ciencias de la Educación, año 10, número 9, enero a diciembre de 2014. Páginas 83-96. ISSN 1851-6297. ISSN EN LÍNEA 2362-3349. LA DIMENSIÓN AMBIENTAL COMO COMPONENTE DE LA EDUCACIÓN SUPERIOR, LA Ciencia y la tecnología en Cuba. Lourdes Ruiz GutiérRez.

y de las regiones. Se han llevado a cabo experiencias previas, las cuáles indican que existe una base previa de información, que por supuesto requiere ser complementada. Cuba fue considerada por la organización internacional Word Wildlife Foundation (WWF) en un informe del 2006 como el único país del mundo en avance hacia la sostenibilidad, al conjugar una mejora medio ambiental, el logro de objetivos sociales y una tendencia no consumista, con un moderado crecimiento económico. Este precedente contribuye a estimular que se realicen estudios rigurosos en nuestro país de los avances hacia el desarrollo sostenible. Los riesgos asociados al cambio climático en regiones vulnerables, acentúan el interés en evaluar la sostenibilidad en determinados territorios.

En diversas actividades de investigación, formación de postgrado, tesis de maestría y de doctorado, el tema del desarrollo sostenible ha sido abordado en el Instituto Superior de Tecnologías y Ciencias Aplicadas (InSTEC), la Universidad de la Habana y el Instituto Superior Politécnico José Antonio Echeverría.

El presente proyecto está financiado por la Agencia Española de Cooperación Internacional para el Desarrollo (AECID) y por el InSTEC. Aspira a lograr un fortalecimiento de la capacidad institucional para las evaluaciones del desarrollo sostenible, sistematizando la base conceptual y metodológica requerida, la base material y documental y la preparación de un grupo de especialistas. Se aspira a obtener una base metodológica general, complementadas con la ejecución de un grupo de experiencias concretas en municipios, regiones naturales y actividades ramales, que contribuyan a desarrollar metodologías específicas para una evaluación del desarrollo sostenible en las áreas y actividades seleccionadas.

- Proyecto GEO- Cuba

Este proyecto permitió la participación de varias universidades cubanas y constituye una evaluación del medio ambiente cubano donde se definen varios de los programas y acciones aplicadas para alcanzar el desarrollo sostenible.

La evaluación del medio ambiente cubano tuvo como propósito, mostrar a todo el pueblo $-\mathrm{y}$ muy especialmente a los tomadores de decisiones-, la importancia de comprender la estrecha relación que existe entre el medio ambiente y el bienestar humano, y la necesidad de que en todos los casos las políticas ambientales vayan a la par de las políticas económicas y del desarrollo emergente y que den respuesta a los principales problemas ambientales a través de acciones de remediación, mitigación y adaptación, como alternativa indispensable para el desarrollo sostenible en nuestro país. También destacó la importancia de la labor científica para el logro de la sosteniblidad, que transita por la identificación de problemas y prioridades ambientales, la elaboración de propuestas de soluciones y su apoyo a la incorporación de estas a la adopción de políticas.

Como metodología de investigación, se aplicó el enfoque ecosistémico en los planes de desarrollo, en lugar del enfoque sectorial. A través de cinco 
Revista de la Escuela de Ciencias de la Educación, año 10, número 9, enero a diciembre de 2014. Páginas 83-96. ISSN 1851-6297. ISSN EN LÍNEA 2362-3349. LA DIMENSIÓN AMBIENTAL COMO COMPONENTE DE LA EDUCACIÓN SUPERIOR, LA Ciencia y la tecnologia en Cuba. Lourdes Ruiz GutiérRez.

ejemplos concretos, correspondientes a diferentes tipos de ecosistemas (montañoso, marino-costero, fluvial, fluvial-marino y urbano) ubicados en zonas de importancia ecológica, social y económica del país, se muestra cómo las interrelaciones que se establecen entre las actividades humanas y el sistema ambiental de esas zonas, alteran la capacidad del ecosistema de proporcionar productos y servicios clave para el bienestar humano y cómo las evaluaciones ambientales con un enfoque sistémico pueden generar respuestas integrales a los servicios del mismo, basadas en las interrelaciones entre las presiones, el Estado y los impactos y, por tanto, contribuir a preservar y elevar el bienestar humano.

El proyecto es participativo, ya que se realizó durante casi dos años un proceso de consultas y donde intervinieron más de 70 universidades e instituciones científicas y no gubernamentales y cerca de 130 profesores, investigadores y especialistas. Asimismo, se destaca cómo las universidades y los centros de ciencia han contribuido a la rehabilitación y restauración de ecosistemas degradados, los estudios territoriales de peligro, vulnerabilidad y riesgo, los diagnósticos ambientales municipales, las evaluaciones de impactos de los desastres y el cambio climático; así como a las medidas de mitigación y adaptación, el manejo integrado de zonas costeras, cuencas hidrográficas y ecosistemas montañosos, la incorporación de la dimensión ambiental a las nuevas inversiones desde la etapa de diseño y los programas para la adecuada gestión de recursos vitales como el agua y los suelos, entre otros avances (Fernández, A; Pérez, R; Garea, B; 2007).

Los impactos negativos sobre el medio ambiente que inciden negativamente sobre la población, se deberán continuar abordando por la comunidad universitaria y científica como problemas transversales y territoriales más que sectoriales, en especial, aquellos relacionados con la contaminación, el inadecuado manejo, tratamiento y disposición final de los desechos sólidos y las aguas residuales. Igualmente deberá prestarse especial atención a la mitigación de posibles impactos derivados de la industria, la intensificación en la producción de alimentos, el incremento en la producción de petróleo y sus derivados y gas, la explotación minera, la acuicultura y el turismo en ecosistemas frágiles, entre otros. En esta línea de acción la educación superior cubana tiene un enorme reto para contribuir a alcanzar el desarrollo sostenible dentro de un programa social y económico que se propone también la sostenibilidad.

\section{Conclusiones}

Se evidencia dentro de los resultados que se han fortalecido los mecanismos de integración del sistema educativo en la educación superior, así como los ejes metodológicos y científicos que lo sustenta en su estrecha vinculación al sistema de ciencia, innovación tecnológica y medio ambiente de Cuba. 
Revista de la Escuela de Ciencias de la Educación, año 10, número 9, enero a diciembre de 2014. Páginas 83-96. ISSN 1851-6297. ISSN EN LÍNEA 2362-3349. LA DIMENSIÓN AMBIENTAL COMO COMPONENTE DE LA EDUCACIÓN SUPERIOR, LA Ciencia y la tecnología en Cuba. Lourdes Ruiz GutiérRez.

Los resultados fundamentales contribuyen al perfeccionamiento de la gestión ambiental y a propiciar cambios de actitudes y prácticas hacia el medio ambiente, lo que fortalecerá la cultura general e integral de todos los ciudadanos.

Es importante, continuar estrechando los vínculos entre las universidades y las organizaciones de la sociedad cubana, que directa o indirectamente trabajan a favor del medio ambiente y el desarrollo sostenible en el país.

Se deben continuar priorizando en las universidades investigaciones en áreas tales como: la educación ambiental, la introducción de los enfoques de la sostenibilidad en todos los sectores de la sociedad y la economía, la mitigación de la contaminación y otros impactos ambientales del sector productivo y el desarrollo local, entre otros.

\section{Referencias Bibliográficas}

- Academia de Ciencias de Cuba (s.f.) Informe de la Academia de Ciencias de Cuba. Obtenido el día 23 de enero 2013 desde la base de datos: http://www.acc.cu. La Habana.

- CITMA, (2005). Informes sobre la Política Científica en Cuba. Ministerio de Ciencia, Tecnología y Medio Ambiente. Dirección de Política Científica. La Habana: Documentos de Archivos de CITMA.

- $\quad$ CITMA, (2007). Estrategia Ambiental Nacional 2007-2010. Ministerio de Ciencia, Tecnología y Medio Ambiente. Dirección de Medio Ambiente. La Habana: Editorial Academia.

- $\quad$ CITMATEL (2007). Resultados de la Ciencia en Cuba. Obtenido el día 28 de enero 2013 desde la base de datos: http://www.citmatel.cu. La Habana

- Fernández A., Pérez R., Garea B, (eds.) (2007). Informe GEO- Cuba 2007. Evaluación del Medio Ambiente Cubano Agencia de Medio Ambiente y Programa de Naciones Unidas para el Medio Ambiente. La Habana: Editorial Academia.

- Gutiérrez R. L., Céspedes, D. G., Cazorla, L. L. (2014). Consideraciones sobre indicadores de desarrollo sostenible en las universidades. Deserción, calidad y reforma universitaria. Apuntes para el debate. Revista Cuadernos del Contrato Social por la Educación, No. 10. Ecuador. 1(2), 88-100. Editorial Cuadernos del Contrato Social por la Educación.

- $\quad$ López, C., Iturralde, M., Claro, R., Ruiz, L., Cabrera, G., Molerio, L. (2002). Introducción al conocimiento del medio ambiente. Suplemento. Universidad para todos. Editorial Academia. Habana.

- O'Reilly, V., Hernández, R. A. B., Gutiérrez, L. R. (2010). Las tecnologías del concreto en su ciclo de vida. Concreto y cemento: Investigación y desarrollo. México 1(2), 42-47.

- PCC (2011). Resolución sobre los Lineamientos de la Política Económica y Social del Partido y la Revolución en Cuba. Partido Comunista de Cuba. La Habana: Editora Política.

- PNUD (2003). Investigación sobre Ciencia, Tecnología y Desarrollo Humano en Cuba. Ciencia y Tecnología al Servicio del Desarrollo Humano en Cuba. Programa de Naciones Unidas para el Desarrollo, La Habana. Obtenido el día 3 de enero 2012 desde la base de datos: http://www.pnud.org.cu.

- Ruiz, L. (1999). La Evaluación de Impacto Ambiental de las Construcciones Turísticas en la Cayería Norte y otras zonas costeras de Cuba. Facultad de Arquitectura. Tesis en opción al grado científico de Doctor en Ciencias Técnicas. 